\title{
Dentigerous cyst associated with impacted three, a rare entity.
}

\author{
Dr. Swapnil Moghe ${ }^{1}$, Dr. Ajay Pillai ${ }^{2}$, Dr. Deepesh Gupta ${ }^{3}$, Dr. Seema chauhan ${ }^{4}$ \\ , Dr. Shubha kumar ${ }^{5}$,Dr. Anjali Moghe ${ }^{6}$ \\ I'Reader,Department Of Oral \& Maxillofacial Surgery, Peoples Dental Academy, Peoples University, Bhopal, \\ India.) \\ ${ }^{2}$ (Reader, Department Of Oral \& Maxillofacial Surgery, Peoples Dental Academy, Peoples University, Bhopal, \\ India) \\ ${ }^{3}$ (Associate Professor, Govt. Dental college,Raipur, India) \\ ${ }^{4}$ (Consultant Dental Surgeon, Sevasadan hospital,Bhopal) \\ ${ }^{5}$ (Consultant Dental Surgeon, Bhopal) \\ ${ }^{6}$ (Ex.Lecturer, Department Of Oral Medicine \& Radiology, Peoples Dental Academy, Bhopal, India)
}

\section{Introduction}

A dentigerous cyst is a developmental cyst of odontogenic origin, which apparently develops by accumulation of fluid between the reduced enamel epithelium and the crown of an unerupted tooth, with consequent expansion of tooth follicle. It may be associated with crown of unerupted teeth, odontomas, or supernumerary teeth. Ninety-five percent of dentigerous cysts are associated with permanent dentition whereas only $5 \%$ are with supernumerary teeth.

Occurrence of a dentigerous cyst with an erupted anterior tooth is exceptionally rare and seldom reported in literature. We report an unusual presentation of a dentigerouscyst associated with an unerupted maxillary central incisor, lateral incisor \&canine associated with over-retained deciduous teeth. A detailed clinical and radiographic examination augmented its accurate histopathological diagnosis.

\section{Case Report}

Herein, we report a case of dentigerous cyst associated with impacted inverted supernumerary teeth in anterior maxilla in an 18-year-old male (Figure 1). The pre-operative clinical picture depicts retained deciduous teeth (Figure 2). The patient was treated surgically by enucleation of total cyst (Figure 3) and surgical extraction of impacted three\& over-retained three deciduous teeth (Figure 4), under general anesthesia and were subsequently confirmed histopathologically. This case is unique \& has rare presentation, as it was associated with over-retained three deciduous teeth \& impacted three teeth. Not many cases have been reported in the literature for the same.

Under all aseptic conditions, the patient was intubated under G.A. \&local anesthetic was infiltrated around anterior maxillary region on the right side. All over-retained deciduous teeth were removed. A crevicular incision was placed from the right side central incisor till first molar with bilateral releasing incisions. A full thickness muco-periosteal flap was raised \& through the opened window, the cystic lining was removed. The surgical site was irrigated with betadine \& saline. Hemostasis was achieved \& closure was done with 3-0 black silk.

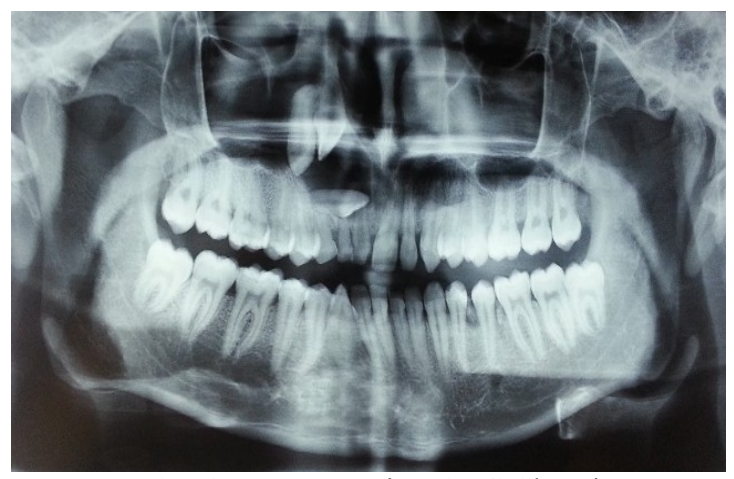

Fig. 1: Pre-operative OPG Showing Over-retained A,B,C.

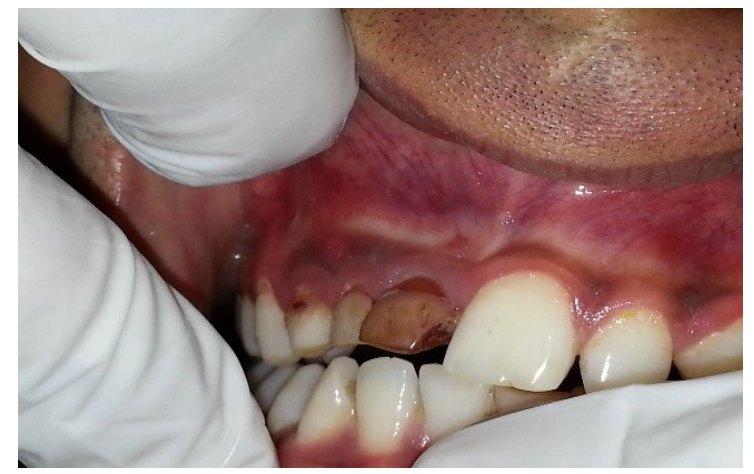

Fig. 2:Pre-operative clinical picture showing Impacted 11,12,13.Over -retained A,B,C. 


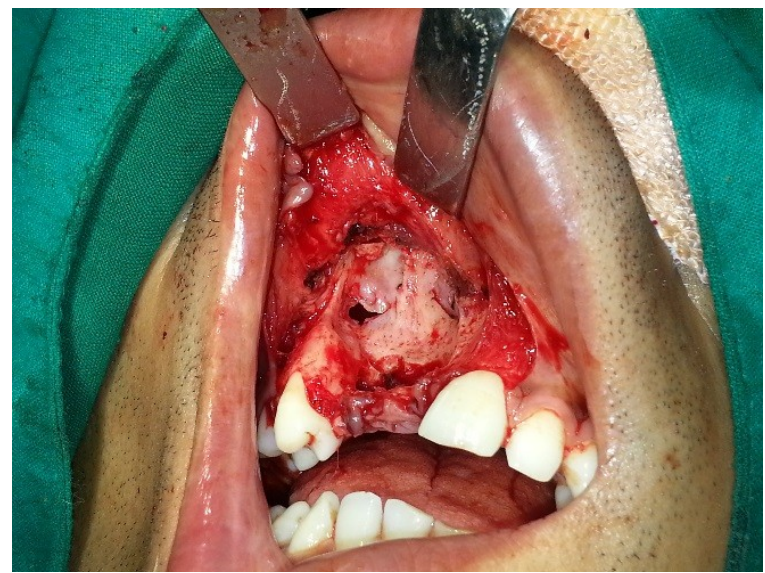

Fig.3: Surgical picture after enucleation.

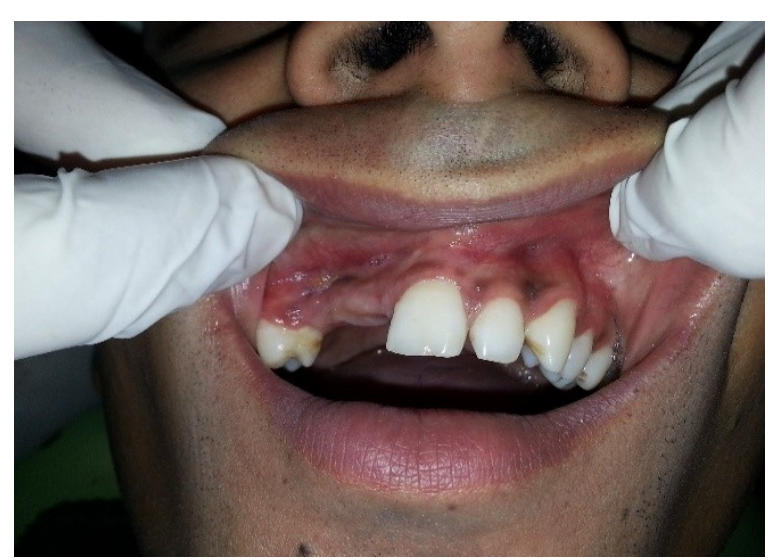

Fig. 5:Post-operative clinical picture Showing good healing.

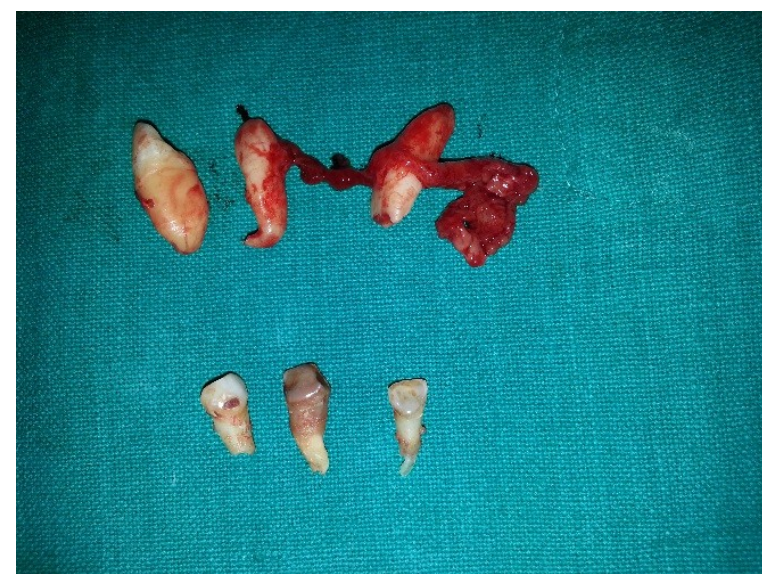

Fig. 4: Cystic lining along with extracted teeth.

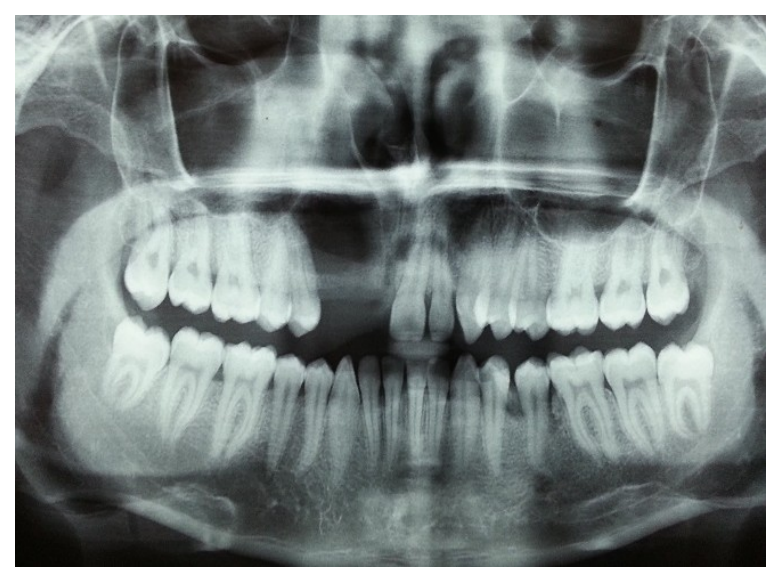

Fig. 6: Post-operative OPG depicting absence of pathology.

\section{Treatment \& follow up:}

The patient was followed up for 1 year(Figure 5)\& the result was uneventful with no signs of recurrence were evident. The postoperative OPG showed good healing at the surgical site. (Figure 6)

\section{Discussion:}

The dentigerous cyst, also known as the follicular cyst, as it arises from the follicle of a tooth germ or unerupted tooth or rarely an odontome, enclosing the same within it. It develops by the cystic degeneration of the epithelial component of the enamel organ and the resultant fluid accumulation between the reduced enamel epithelium and enamel of the tooth ${ }^{1}$.

Main's gave a theory (1970) to explain this phenomena: The impacted tooth exerts pressure on the follicle which obstructs the venous outflow and induces rapid transudation of serum across the capillary walls. The increased hydrostatic pressure exerted by this pooling of fluid causes separation of crown from the follicle with or without reduced enamel epithelium. The osmolality of the cyst fluid is modified by increased permeability to glycosaminoglycan's like hyaluronic acid, heparin \& chondroitin sulphate which causes expansile growth rapidly (Browne \& Smith et $\mathrm{al}^{2}, 1980$ ).

Radio graphicallydentigerouscysts may be unilocular or multilocular with well-defined margins. It may or may not encroach the adjacent vital structures like maxillary antrum, inferior alveolar neurovascular bundle, etc. Treatment of dentigerous cyst depends on size, location, disfigurement \& often requires variable bone removal to ensure total removal of the cyst, especially in cases of large ones ${ }^{3}$.Thus treatment for dentigerous cysts is surgical removal ${ }^{4}$. Because of the potential for occurrence of an odontogenickeratocyst, adenomatoidodontogenic tumour ${ }^{5}$ or the development of an ameloblastoma or muco-epidermoid carcinoma, all such lesions, when removed, should be submitted for histo-pathological evaluation. Surgeries are further complicated by readily displacement of tooth into the maxillary sinus, soft tissue of the infra-temporal space, fractures and paraesthesias ${ }^{6}$. Interdisciplinary decisions with endodontists and orthodontists like salvaging the tooth or extraction are to be made further, so that to maintain the overall harmonious occlusal relationships and taking age into considerations. 
Removable prosthesis is commonly required as a splint after the enucleation of the lesion, where primary closure is not possible.

We report a case of dentigerous cyst associated with over-retained deciduous teeth \& impacted central incisor, lateral incisor \& canine, an rare entity.

\section{Conclusion}

Recurrence of dentigerous cyst is rare and could be due to the residual fragments of the cystic lining. To prevent the development of a dentigerous cyst and to avoid unwanted effects on adjacent teeth, early detection consisting of a thorough clinical and radiographic examination is necessary for accurate diagnosis and proper treatment planning.

\section{References:}

[1]. Shear M, Speight PM. Dentigerous cyst. Cysts of the Oral \& Maxillofacial region. $4^{\text {th }}$ ed. USA: Blackwell publishing Professional; 2007. P. 59-75.

[2]. Browne RM, Smith AJ: Pathogenesis of odontogenic cysts. In: Investigative Pathology of the OdontogenicCyst. CRC Press Boca Raton, 1991;P. 88-109.

[3]. Primosch RE. Anterior supernumerary teeth: assessment and surgical intervention in children. Pediatric Dentistry, 1981; 3: 204-21.

[4]. Kessler HP, Kraut RA. Dentigerous cyst associated with an impacted mesiodens .General Dentistry,1989; 37(1):47-9.

[5]. Sandu S, Narang K, Jwanda M. Adenomatoidtumour associated with dentigerous cyst of maxillary antrum a rare entity. J Oral Maxillofacial Pathology 2010; 14:24-8.

[6]. Salman L Problems of impacted teeth 1958; 11: 1357-71. 